\title{
CTLA-4 Gene Polymorphism in Relation to Thyroid Disorders in Patients with Chronic Hepatitis C Under Interferon Alpha Therapy
}

\author{
${ }^{1}$ Salwa M. Abo El-Khair, ${ }^{2}$ Abd El-Hameed A. Metwali, \\ ${ }^{3}$ Mohamed Abd El-Maksoud and ${ }^{3}$ Tarek Besheer \\ ${ }^{1}$ Department of Medical Biochemistry, \\ ${ }^{2}$ Departments of Internal Medicine, Diabetes and Endocrinology Unit, \\ ${ }^{3}$ Department of Tropical Medicine, \\ Faculty of Medicine, Mansoura University, Egypt
}

Received 2012-05-07, Revised 2012-08-01; Accepted 2012-08-17

\begin{abstract}
Thyroid autoimmunity and dysfunction have reported as side effects of interferon- $\alpha$ treatment. The CT60 and exon 1+49 A/G polymorphisms in the Cytotoxic T-Lymphocyte Antigen 4 (CTLA-4) gene have linked to susceptibility to autoimmune disease. The aim of this research was to analyze the frequencies of CTLA-4 CT60 and $+49 \mathrm{~A} / \mathrm{G}$ polymorphisms and evaluate both polymorphisms as indicators of thyroid disorder susceptibility in chronic HCV Egyptian patients under interferon therapy. This study was carried out on 114 chronic HCV patients under combined therapy of interferon- $\alpha$ and Ribavirin. From them, 60 chronic HCV patients without thyroid disorder were considered the control group. The other 54 patients were having thyroid disorder either hypothyroidism $(\mathrm{N}=35)$ or Grave's Disease $(\mathrm{GD})(\mathrm{N}=19)$. For all subjects, the genotypes of CTLA-4 gene CT60 and $+49 \mathrm{~A} / \mathrm{G}$ polymorphisms were studied using RFLP technique. For +49 A/G polymorphism, the genotypes frequencies in controls were: AA (30), AG (31.7) and GG (38.3\%). Whereas, in hypothyroidism patients, the AA, AG and GG genotypes frequencies were (20), (57.1\%) and (22.9\%) respectively, while in grave's disease, the AA, AG and GG genotypes frequencies were (26.3 (57.9) and (15.8\%) respectively. The AG genotype was significantly associated with thyroid disease. As regards CT60 polymorphism, the genotypes frequencies in controls were: CC (38.3), CT (35) and TT (26.7\%). Whereas, in hypothyroidism patients, the CC, CT and TT genotypes frequencies were (60), (20) and (20\%) respectively, while in grave's disease, the CC, CT and TT genotypes frequencies were (68.4), (15.8) and (15.8\%) respectively. The CC genotype was significantly associated with thyroid disorders. CTLA-4 gene $+49 \mathrm{~A} / \mathrm{G}$ and CT60 polymorphisms confer susceptibility to autoimmune thyroid disorder and confirm usefulness of CTLA-4 genotyping in predicting thyroid disorder in chronic HCV patients under interferon therapy.
\end{abstract}

Keywards: Cytotoxic T-Lymphocyte Antigen 4, +49A/G, CT60, RFLP, Autoimmune Disease, Hepatitis C Virus

\section{INTRODUCTION}

Hepatitis C Virus (HCV) infection is a potentially life-threatening disease as $75 \%$ of affected patients with acute infection develop chronic disease with a high risk of cirrhosis and hepatocellular carcinoma (Carella et al., 2004). Treatment of hepatitis $C$ with pegylated interferon $\alpha$ plus Ribavirin as a Standard Of Care (SOC) for the management of this disease, induces a biochemical (persistent normalization of alanine-aminotransferase) and virological (sustained negativity for Hepatitis $\mathrm{C}$ Virus [HCV] RNA) response and leads to eradication of the virus in less than $60 \%$ of patients (Missiha et al., 2008). Adverse effects of interferon (IFN) treatment Egypt Tel: 002/01117488887 
include systemic and organ-specific pathological changes, many of them being the consequences of immune enhancement or immune dysregulation induced by IFN itself (Carella et al., 2004). The main effect of IFN- $\alpha$ on the immune system is the enhancement of cell cytotoxicity, which is important for antineoplastic and antiviral activity (Carella et al., 2004).

The widespread effects of IFN $\alpha$ on the immune system may be important for inducing thyroid disease because peripheral features of systemic immune involvement have been described in patients with thyroid autoimmunity (Ciampolillo et al., 2003). In addition to the systemic effects, IFN $\alpha$ may have direct effects on the thyroid gland by modulating the aberrant expression of major histocompatibility antigens on thyroid cells (Carella et al., 2004) and favoring a cytokine microenvironment, which may lead to the immunemediated damage of thyroid tissue (Wang et al., 2002a). Furthermore, IFN $\alpha$ has direct inhibitory effects on thyroid hormone synthesis, release and metabolism (Corssmit et al., 1995). However, the critical point is to understand how a generalized activation of the immune system, induced by the cytokine treatment, may result in an organ-specific involvement of the thyroid gland (Carella et al., 2004). A genetic predisposition to thyroid autoimmune disease is probably necessary for the development of thyroid disease in patients treated with IFN $\alpha$ (Carella et al., 2004).

The Cytotoxic T-Lymphocyte Antigen 4 (CTLA-4) gene on human chromosome $2 \mathrm{q} 33$ is one of the candidate genetic markers (Weng et al., 2005). The most remarkable function of the CTLA-4 gene is downregulation of the humoral immune response. CTLA-4 is an immune regulatory molecule expressed on the surface of activated $\mathrm{T}$ lymphocytes and a key inhibitor of $\mathrm{T}$ cell activation (Kavvoura et al., 2007).

Genetic polymorphisms in the human genome are an important component of genotypic variability including one's immune status (Sun et al., 2009). The CTLA-4 gene contains several Single Nucleotide Polymorphisms (SNPs) that may influence gene expression, cause amino acid substitution and alter mRNA splicing, which may manipulate T-cell activation and, eventually, modulate host immune status. Although functional alterations resulting from CTLA-4 polymorphisms seem to be subtle, the biological outcomes are significant. The SNPs in the Cytotoxic T-Lymphocyte Antigen 4 (CTLA-4) gene have been linked to susceptibility to autoimmune disease (Sun et al., 2009).
Several polymorphic sites in the CTLA-4 gene (i.e, $\mathrm{A} / \mathrm{G}$ polymorphism in exon $1+49$ (Kouki et al., 2000), $\mathrm{C} / \mathrm{T}$ polymorphism in the promoter -318 (Park et al., 2000), microsatellite (AT)n repeat in the 3'-untranslated region (UTR) of exon 4 (Kouki et al., 2002) and 3 SNPs in the 6.1-kb 3' non-coding region, CT60, JO31 and JO30) have been reported to be associated with the organ-specific autoimmune disorders (Ueda et al., 2003; Orozco et al., 2004; Torres et al., 2004) in several racial groups (Furugaki et al., 2004; Weng et al., 2005). Among them, CT60, C/T polymorphism in the promoter -318 and exon $1+49$ A/G SNPs were the markers most associated with autoimmune endocrinopathies (Vaidya and Pearce, 2004).

According to prior studies on the association of the development of various autoimmune diseases and the CTLA4 gene, the $+49 \mathrm{~A} / \mathrm{G}$ polymorphism in CTLA4 exon 1 has been reported to be involved in the development of autoimmune diseases (Jung et al., 2009) including Graves' disease (Yanagawa et al., 1997), Hashimoto thyroiditis (Vaidya and Pearce, 2004), a portion of Addison's disease and rheumatoid arthritis (Vaidya et al., 2002). A significant association of Type I Diabetes (T1D) with the CTLA4 polymorphisms was reported by Jung et al. (2009).

The CT60 in the 3'UTR region of CTLA4 gene is the most promising locus for the autoimmune thyroid disease (Ban et al., 2005; Daroszewski et al., 2009). The meta-analysis study of Kavvoura et al. (2007), shows consistent associations between Graves' disease and Hashimoto thyroiditis with CT60. This association crosses ethnic barriers. Although they cannot identify a single etiological polymorphism, their study clarifies the important role of the CTLA-4 locus in determining the risk of autoimmune thyroid diseases.

\subsection{Aim of the Study}

Many studies describe the association between a polymorphism of the CTLA-4 gene and autoimmune disease, however, none of these previous studies was carried out in HCV patients under interferon therapy. So, the aim of this research is to analyze the frequencies of CTLA-4 CT60 and exon 1+49 A/G polymorphisms in chronic HCV Egyptian patients under interferon therapy and to evaluate these two polymorphisms of CTLA-4 gene as indicators of thyroid disorder susceptibility in chronic HCV patients.

\section{MATERIALS AND METHODS}

\subsection{Subjects}

Fifty four patients with autoimmune thyroid disorders (39 females, 15 males) were selected from a 
series of patients receiving combined therapy of interferon- $\alpha$ and Ribavirin for treatment of chronic HCV at outpatient clinic of tropical medicine at Mansoura University Hospital. The diagnosis of grave's disease was based on clinical features of hyperthyroidism and laboratory results including (a) raised serum free thyroxine (T4) or free 3,5,3'-triiodothyronine (T3) levels, suppressed serum thyrotropin (TSH) levels, (b) the presence of detectable thyrotropin receptor autoantibodies, antimicrosomal and/or antithyroglobulin antibodies and (c) increased radioactive technetium uptake. The diagnosis of hypothyroidism was based on clinical features of hypothyroidism and laboratory results including low serum free T4 or free T3 with elevated serum level of $\mathrm{TSH}$, beside presence of detectable, antimicrosomal and/or antithyroglobulin antibodies. In addition, 60 patients, matched for age and sex, with chronic HCV receiving combined therapy of interferon- $\alpha$ and Ribavirin, with no clinical features of thyroid disorders and normal free T3, free T4 and TSH levels with negative thyrotropin receptor autoantibodies, antimicrosomal and/or anti-thyroglobulin antibodies were also included as controls.

Patients having any of the following diseases were excluded from the current study: diabetes mellitus, chronic renal failure, coronary artery disease, end stage liver disease, malignancy, positive serum Antinuclear Antibody (ANA). Patients and controls showing clinical and/or biochemical evidence of cortisol excess or deficiency, hypogonadism, growth hormone deficiency and pituitary adenoma were also excluded.

All subjects were informed of the purpose of the study and informed consents were obtained .The study was approved by the ethical committee of Mansoura University.

\subsection{Biochemical Investigation}

All subjects were instructed to fast for at least $12 \mathrm{~h}$. A blood sample was withdrawn. $5 \mathrm{mLs}$ were delivered to centrifuge tubes containing K2EDTA (stored as EDTA anti-coagulated blood sample at $-30^{\circ} \mathrm{C}$ for DNA extraction). Also, plasma samples were prepared to obtain plasma for measurement of plasma CTLA-4 levels. Another $5 \mathrm{~mL}$ blood sample was allowed to clot for 15 min and centrifuged at $7000 \mathrm{rpm}$ for $10 \mathrm{~min}$ for serum separation to determine: liver enzymes, serum albumin, free T3, free T4, TSH, thyrotropin receptor autoantibodies, antimicrosomal and/or antithyroglobulin antibodies.

\subsection{DNA Extraction}

Genomic DNA was extracted from EDTAanticoagulated peripheral blood leucocytes using QIA amp DNA Blood Mini Kit supplied by Qiagen $\mathrm{GmbH}$ (Cat, No. 51104, Hiden, Germany) (Schur et al., 2001). The average DNA concentration $\left(0.127 \pm 0.005 \mu \mathrm{g} \mu \mathrm{L}^{-1}\right)$ was determined from absorbance at $260 \mathrm{~nm}$ (Jenway, Genova Model, UK). All samples had a 260/280 nm absorbance ratio between 1.6 and 1.79 . The integrity of the DNA was checked by electrophoresis on $0.8 \%$ agarose gel stained with ethidium bromide.

\subsection{Genotyping of $\mathrm{A} / \mathrm{G}$ Polymorphism at Position 49 in Exon 1 of the CTLA4 Gene (Jung et al., 2009) \\ 2.5. Polymerase Chain Reaction (PCR)}

The primers sequences used for DNA amplification are as follow: 5'-GCTCTACTTCCTGAAGACCT-3' (forward) and 5'-AGTCTCACTCACCTTTGCAG3 '(reverse) (Donner et al., 1997). PCR was carried out in 50 micro liters final reaction volume using Ready Mix (RED. Taq-PCR Reaction Mix) (purchased from Sigma Aldrich, Saint Louis, USA). The following mixture was prepared for each sample: $25 \mu \mathrm{L}$ RED-Taq PCR reaction Mix $(1 \times), 1 \mu \mathrm{L}(20$ pmole $)$ of forward primer, $1 \mu \mathrm{L}(20$ pmole) of reverse primer, $2 \mu \mathrm{L}(200 \mathrm{ng})$ of genomic DNA and $21 \mu \mathrm{L}$ of double distilled deionizer water. This mix was put in a thin wall PCR micro centrifuge tube and gently centrifuged to collect all components to the bottom of the tube. Then $50 \mu \mathrm{L}$ mineral oil was added to prevent evaporation. Amplification was performed in a Thermal Cycler (Minicycler-PTC-150) using the following program: initial $5 \mathrm{~min}$ denaturation at $94^{\circ} \mathrm{C}$ followed by 30 cycles of denaturation at $94^{\circ} \mathrm{C}$ for 30 sec, annealing at $55^{\circ} \mathrm{C}$ for $30 \mathrm{sec}$, extension at $70^{\circ} \mathrm{C}$ for $30 \mathrm{sec}$ and a final extension for $7 \mathrm{~min}$ at $70^{\circ} \mathrm{C}$. The resulting PCR product was $162 \mathrm{bp}$ in length.

Amplified samples were digested with the specific restriction enzyme BbvI (New England BioLabs, Beverly, MA, U.S.A.) at $37^{\circ} \mathrm{C}$ for $2 \mathrm{~h}$, electrophoresed on a $3 \%$ agarose gel for $60 \mathrm{~min}$, stained with ethidium bromide, visualized via Light UV Transilluminator (Model TUV-20, OWI Scientific, Inc. 800 242-5560, France) and photographed and evaluated. A 162 bp band was determined to be the A allele and the $88 \mathrm{bp}$ and 74 bp bands were determined to be the $G$ allele (Fig. 1).

\subsection{Genotyping of CTLA-4 C/T-60 Single Nucleotide Polymorphism (SNP) in the 3, Untranslated Region (Wang et al., 2007a)}

Polymerase Chain Reaction (PCR): The primers sequences used for DNA amplification are as follow: forward primer 5'- GAGGTGAAGAACCTGTGTTAAA 
-3 and reverse primer 5' ATAATGCTTCATGAGTCAGCTT -3'. PCR was carried out in 50 microliters final reaction volume using ReadyMix (RED. Taq-PCR Reaction Mix) (purchased from Sigma Aldrich, Saint Louis, USA). The following mixture was prepared for each sample: $25 \mu \mathrm{L}$ RED-Taq PCR reaction Mix (1X), $1 \mu \mathrm{L}(20$ pmole) of forward primer, $1 \mu \mathrm{L}$ (20 pmole) of reverse primer, $2 \mu \mathrm{L}(200$ ng) of genomic DNA and $21 \mu \mathrm{L}$ of double distilled deionized water. This mix was put in a thin wall PCR microcentrifuge tube and gently centrifuged to collect all components to the bottom of the tube. Then $50 \mu \mathrm{L}$ mineral oil was added to prevent evaporation. Amplification was performed in a Thermal Cycler (Minicycler-PTC-150) using the following program: initial $5 \mathrm{~min}$ denaturation at $94^{\circ} \mathrm{C}$ followed by 30 cycles of denaturation at $94^{\circ} \mathrm{C}$ for $30 \mathrm{sec}$, annealing at $55^{\circ} \mathrm{C}$ for $30 \mathrm{sec}$, extension at $72^{\circ} \mathrm{C}$ for $30 \mathrm{sec}$ and a final extension for $5 \mathrm{~min}$ at $72^{\circ} \mathrm{C}$. The resulting PCR product was $178 \mathrm{bp}$ in length.

Amplified samples were digested with 10 units of the specific restriction enzyme HpyCH4IV (New England-BioLabs, Beverly, MA, U.S.A.) at $37^{\circ} \mathrm{C}$ for $2 \mathrm{~h}$, electrophoresed on a $3 \%$ agarose gel for $60 \mathrm{~min}$, stained with ethidium bromide, visualized via light UV Transilluminator (Model TUV-20,OWI Scientific, Inc.800 242-5560, France) and photographed and evaluated. The restriction enzyme HpyCH4 IV cuts the 178 Base Pair (BP) PCR product only if the $\mathrm{C}$ allele is present at this site, resulting in fragments of 107 and 71 bp. The C-60 allele yielded two bands of 107 and $71 \mathrm{bp}$ and the T-60 allele yields a band of 178 bp (Fig. 2).

\subsection{Estimation of Plasma CTLA-4 Levels}

Quantitative determination of plasma CTLA-4 levels was performed by BlueGene ELISA Kits, Life Sciences Advanced Technologies, Inc. 2900 72nd St. N. Saint Petersburg, FL 33710, USA. Cat no. E01C0628. This assay employs the quantitative sandwich ELISA technique which measures CTLA-4 in plasma. It was performed according to the manufacturer's instructions. The absorbance of each sample was read on plate ELISA reader (Tecan, Sunrise Absorbance reader) at $450 \mathrm{~nm}$ wavelength.

\subsection{Thyroid Profile Estimation}

ELISA kits were used for estimation of Free T3 (Diagnostic Automation, INC. 23961 Craftsman Road, Suite D/E/F, Calabasas, CA 91302, Cat \# $3148 Z$ ), free T4 (Diagnostic Automation, INC.23961 Craftsman Road, Suite D/E/F, Calabasas, CA 91302, Cat \# 3146Z ), TSH ((Diagnostic Automation, INC. 23961 Craftsman Road, Suite D/E/F, Calabasas, CA 91302, Cat \# 3122Z ).

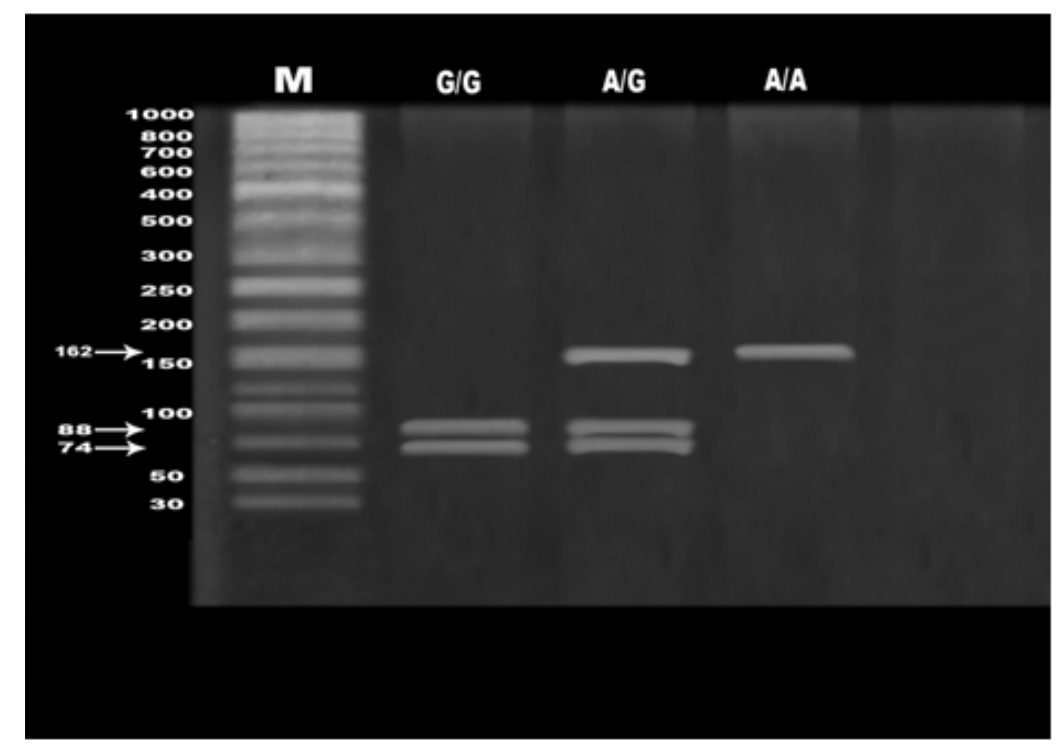

Fig. 1. Agarose gel electrophoresis analysis of the $+49 \mathrm{~A} / \mathrm{G}$ polymorphism in CTLA-4 gene after BbvI digestion. The AA genotype is presented by one single band at $162 \mathrm{bp}$. (lane 4 ) and GG genotype is presented by two bands at 88 and $74 \mathrm{bp}$. (lane 2). While AG genotype is presented by three bands: 162,88 and $74 \mathrm{bp}$. (lane 3). lane (1) represents the molecular marker (DNA molecular weight marker, purchased from promega tecnical service, catalog \#G3161 


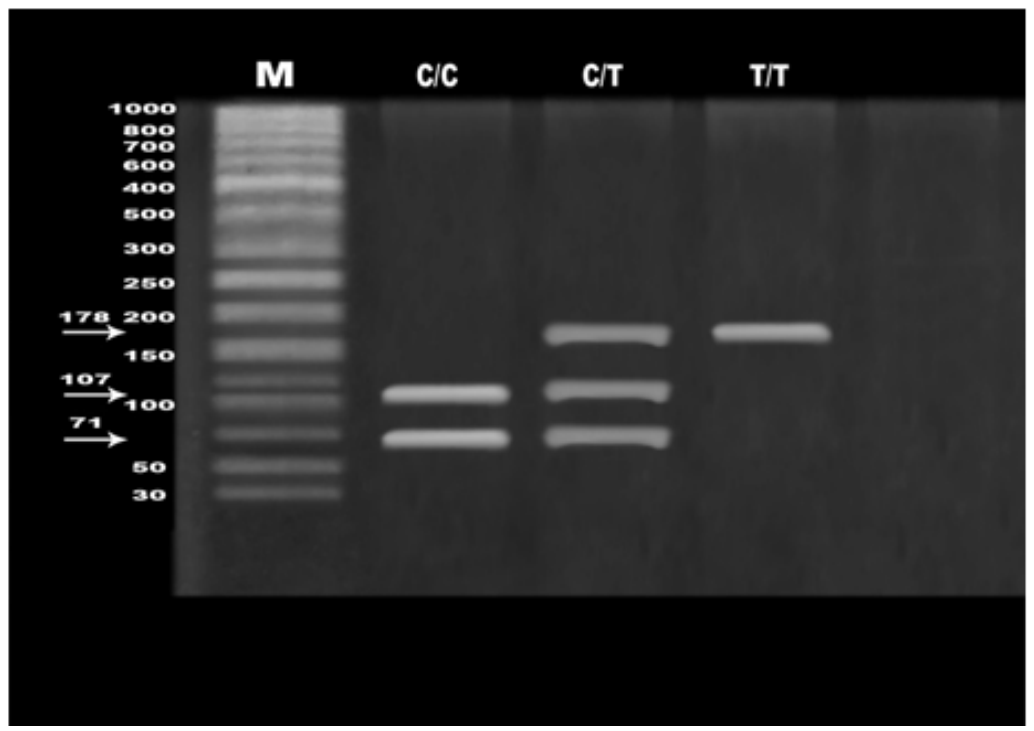

Fig. 2. Agarose gel electrophoresis analysis of the CT60 polymorphism in CTLA-4 gene after HpyCH4IV digestion. The TT genotype produced only one $178 \mathrm{bp}$ band (lane 4), the CC genotype produced 2 small fragments at 107 and $71 \mathrm{bp}$ respectively (lane 2) and the CT genotype produced 3 bands: 178, 107and 71 bp respectively (lane 3 ). Lane (1) represents the molecular marke (DNA molecular weight marker, purchased from promega tecnical service, catalog \#G3161)

Thyrotropin receptor autoantibodies were detected by using ElisaRSRTM TRAb 3rd Generation, RSR Limited Avenue Park Pentwyn Cardiff CF23 8HE United Kingdom (Kamijo et al., 2005). Anti-thyroid microsomal and anti-thyroglobulin antibodies were measured by particle agglutination (Serodia-AMC and Serodia-ATG diagnostic kits, Fujirebio Inc., Tokyo, Japan) and were scored as positive if either was present at titres more than 1/100 (Vanderpump et al., 1995).

\subsection{Lipid Profile Estimation}

Lipid profile estimation was performed using the commercially available kits; Total cholesterol (Allian et al., 1974), triglycerides (Fossati and Prencipe, 1982), HDL-C (Lopeo-Virella et al., 1977) and LDL-C (Friedewald et al., 1972). Serum levels of albumin (Dumas et al., 1997), Alanine transaminase enzyme (ALT) and Aspartate Transaminase enzyme (AST) (Thefeld et al., 1974) were also estimated.

\subsection{Statistical Analysis}

Data were collected, revised, verified then edited on personal computer and then analyzed using the Statistical Package of Social Sciences (SPSS) version 16 for Windows (SPSS, Inc., Chicago, IL, USA). Data were presented using mean and Standard Deviation (SD) for all quantitative values and number of cases (percentage) for qualitative values. The distribution of tested variables was examined with Kolmogorov-Smirnov test for normality. The significance of differences between continuous variables was determined with independent samples t- test for parameters with normal distribution and Mann-Whitney test for non normally distributed variables, as appropriate. Chi- square or Fisher exact test was used for comparison between qualitative variables, as appropriate. One-way Analysis of Variance (ANOVA) was used to examine the mean differences between more than 2 groups distributed normally and Kruskal Wallis test for data not normally distributed, as appropriate. $\mathrm{P}$ values $<0.05$ at confidence interval $95 \%$ were considered significant for all statistical analyses in this study.

\section{RESULTS}

This study was carried out on 114 chronic HCV patients under combined therapy of interferon- $\alpha$ and ribavirin. According to presence of clinical features of thyroid disorders, levels of free T3, free TS and TSH and presence of thyrotropin receptor autoantibodies, antimicrosomal and/or antithyroglobulin antibodies, 60 chronic $\mathrm{HCV}$ patients under combined therapy of 
interferon- $\alpha$ and ribavirin without presence of thyroid disorder were considered as a control group. The other 54 patients were having thyroid disorder either hypothyroidism $(\mathrm{N}=35)$ or grave's disease $(\mathrm{N}=19)$. The demographic, clinical, biochemical and hormonal characteristics of all studied groups were presented in Table 1. For all subjects, the genotypes of CTLA-4 gene CT60 and exon 1+49 A/G polymorphisms were studied.

For CTLA-4 gene exon 1+49 A/G polymorphism, the genotypes frequencies in patients without thyroid disorder (control group) were as follow: AA genotype was $(18 / 60,30 \%)$, AG genotype was $(19 / 60,31.7 \%)$ and GG genotype was $(23 / 60,38.3 \%)$. Whereas, in patients having hypothyroidism, the AA, AG and GG genotypes frequencies were $(7 / 35,20 \%),(20 / 35,57.1 \%)$ and $(8 / 35$,
$22.9 \%$ ) respectively (Table 2 ), while in patients having grave's disease, the AA, AG and GG genotypes frequencies were $(3 / 19,26.3 \%),(11 / 19,57.9 \%)$ and $(3 / 19,15.8 \%)$ respectively (Table 3$)$. The AG genotype was significantly associated with developing either hypothyroidism (odd ratio: 2.6, 95\% CI: 1.1-6.1, $\mathrm{P}=$ 0.02) (Table 2) or grave's disease (odd ratio: $2.97,95 \%$ CI: 1.02-8.6, $\mathrm{P}=0.039$ ) (Table 3). The odd ratio for the risk of hypothyroidism associated with the $\mathrm{G}$ allele was 1.4 (95\% CI 0.6-3.8; $\mathrm{P}=0.3$ ) (Table 2), whereas, the odd ratio for the risk of grave's disease associated with the $\mathrm{G}$ allele was 1.2 (95\% CI 0.4-3.8; $\mathrm{P}=0.5)$ (Table 3). This indicates non significant association of the mutant $\mathrm{G}$ allele with developing thyroid disorder in chronic $\mathrm{HCV}$ patients under interferon $\alpha$ therapy.

Table 1: Demographic, clinical, biochemical and hormonal characteristics of the studied groups

\begin{tabular}{|c|c|c|c|c|c|}
\hline Variables & $\begin{array}{l}\text { Controls }(\mathrm{N}=60) \\
\text { mean } \pm \mathrm{SD}\end{array}$ & $\begin{array}{l}\text { Hypothyroidism }(\mathrm{N}=35) \\
\text { mean } \pm \mathrm{SD}\end{array}$ & $\begin{array}{l}\mathrm{GD}(\mathrm{N}=19) \\
\text { mean } \pm \mathrm{SD}\end{array}$ & $\mathrm{P} 1$ & $\mathrm{P} 2$ \\
\hline \multicolumn{6}{|l|}{ Demographic } \\
\hline Age (years) & $41.8 \pm 7.6$ & $44.3 \pm 12.6$ & $44.8 \pm 11.90$ & 0.200 & 0.200 \\
\hline Gender (male/female) & $36 / 24$ & $7 / 28$ & $8 / 11$ & & \\
\hline Weight (kg) & $73.5 \pm 7.8$ & $81 . .53 \pm 70$ & $61 \pm 5.9000$ & $<0.001$ & $<0.001$ \\
\hline Height $(\mathrm{m})$ & $1.7 \pm 0.1$ & $1.6 \pm 0.04$ & $1.6 \pm 0.100$ & 0.400 & 0.040 \\
\hline $\operatorname{BMI}\left(\mathrm{kg} / \mathrm{m}^{2}\right)$ & $27.01 \pm 3.5$ & $30.2 \pm 2.90$ & $23.3 \pm 2.030$ & $<0.001$ & $<0.001$ \\
\hline \multicolumn{6}{|l|}{ Clinical } \\
\hline $\mathrm{SBP}(\mathrm{mmHg})$ & $131.4 \pm 5.9$ & $132.9 \pm 7.90$ & $133.4 \pm 8.700$ & 0.300 & 0.300 \\
\hline DBP (mmHg) & $79.3 \pm 6.1$ & $79.7 \pm 6.10$ & $78.7 \pm 6.200$ & 0.800 & 0.700 \\
\hline \multicolumn{6}{|l|}{ Biochemical and hormonal } \\
\hline Plasma CTLA-4 (ng/mL) & $23.1 \pm 5.70$ & $79.2 \pm 13.04$ & $79.6 \pm 14.100$ & $<0.001$ & $<0.001$ \\
\hline Total cholesterol (mg/dL) & $187.5 \pm 26.4$ & $202 \pm 25.6$ & $185.8 \pm 44.700$ & 0.010 & 0.800 \\
\hline Triglycerides (mg/dL) & $145.5 \pm 34.6$ & $153.9 \pm 21.4$ & $145.8 \pm 43.500$ & 0.200 & 0.970 \\
\hline HDL-c $(\mathrm{mg} / \mathrm{dL})$ & $43.6 \pm 4.60$ & $42.2 \pm 9.6$ & $40.5 \pm 6.2000$ & 0.300 & 0.050 \\
\hline LDL-c (mg/dL) & $114.8 \pm 22.6$ & $129.02 \pm 25.41$ & $116.2 \pm 40.400$ & 0.006 & 0.900 \\
\hline Free T3 $(\mathrm{pg} / \mathrm{mL})$ & $3 \pm 0.5$ & $1.6 \pm 0.4$ & $6.6 \pm 0.8000$ & $<0.001$ & $<0.001$ \\
\hline Free T4 (ug/dL) & $1.05 \pm 0.2$ & $0.4 \pm 0.1$ & $3.3 \pm 0.6000$ & $<0.001$ & $<0.001$ \\
\hline $\mathrm{TSH}(\mu \mathrm{IU} / \mathrm{mL})$ & $1.8 \pm 0.60$ & $18.8 \pm 9.3$ & $0.02 \pm 0.003$ & $<0.001$ & $<0.001$ \\
\hline
\end{tabular}

Data are given as Mean \pm SD; GD, grave's disease; BMI, body mass index; SBP, systolic blood pressure; DBP, diastolic blood pressure; CTLA-4, cytotoxic T-lymphocyte-associated antigen-4; HDL-c, high density lipoprotein cholesterol; LDL-c, low density lipoprotein cholesterol; T3, triiodothyronine; T4, tetra-iodothyronine; TSH, thyroid stimulating hormone; P1, hypothyroidism vs. controls; P2, hyperthyroidism vs. controls

Table 2. +49 A/G and CT60 polymorphisms genotypes and alleles frequencies in hypothyroidism patients and control subjects

\begin{tabular}{|c|c|c|c|c|}
\hline Variables & Controls $(n=60)$ & Hypothyroid cases $(n=35)$ & Odd ratio(OR) $(95 \% \mathrm{CI})$ & $\mathrm{P}$ \\
\hline \multicolumn{5}{|c|}{ CTLA-4 gene +49 A/G genotypes: } \\
\hline $\mathrm{G} / \mathrm{G}$ n $(\%)$ & $23(38.3 \%)$ & $8(22.9 \%)$ & $0.48(0.19-1.2)$ & 0.09 \\
\hline A/G n $(\%)$ & $19(31.7 \%)$ & $20(57.1 \%)$ & $2.6(1.1-6.1)$ & 0.02 \\
\hline $\mathrm{A} / \mathrm{A} \mathrm{n}(\%)$ & $18(30 \%)$ & $7(20 \%)$ & $0.6(0.2-1.6)$ & 0.20 \\
\hline \multicolumn{5}{|c|}{ CTLA-4 gene +49 A/G alleles: } \\
\hline G allele: $\mathrm{n}(\%)$ & $65(54.2 \%)$ & $36(51.4 \%)$ & $1.4(0.6-3.8)$ & 0.30 \\
\hline A allele: $\mathrm{n}(\%)$ & $55(45.8 \%)$ & $34(48.6 \%)$ & $1.8(0.7-4.5)$ & 0.20 \\
\hline \multicolumn{5}{|c|}{ CTLA-4 gene C/T60 genotypes: } \\
\hline $\mathrm{C} / \mathrm{C}$ & $23(38.3 \%)$ & $21(60 \%)$ & $2.4(1.02-5.7)$ & 0.03 \\
\hline $\mathrm{C} / \mathrm{T}$ & $21(35 \%)$ & $7(20 \%)$ & $0.5(0.2-1.2)$ & 0.09 \\
\hline $\mathrm{T} / \mathrm{T}$ & $16(26.7 \%)$ & $7(20 \%)$ & $0.7(0.3-1.9)$ & 0.30 \\
\hline \multicolumn{5}{|c|}{ CTLA-4 gene C/T60 alleles: } \\
\hline C allele: $n(\%)$ & $67(55.8 \%)$ & $49(70 \%)$ & $1.5(0.5-3.9)$ & 0.30 \\
\hline T allele: $n(\%)$ & $53(44.2 \%)$ & $21(30 \%)$ & $0.4(0.2-0.97)$ & 0.03 \\
\hline
\end{tabular}

OR: Odds Ratio Vs controls; CI: Confidence Interval; Significance was determined using Fisher's exact test 
Salwa M. Abo El-Khair et al. / American Journal of Biochemistry and Biotechnology 8 (3) (2012) 179-194

Table 3. $+49 \mathrm{~A} / \mathrm{G}$ and CT60 polymorphisms genotypes and alleles frequencies in Grave's disease patients and control subjects

\begin{tabular}{|c|c|c|c|c|}
\hline Variables & Controls $(n=60)$ & Grave's disease $(\mathrm{n}=35)$ & Odd Ratio (OR) $(95 \% \mathrm{CI})$ & $\mathrm{P}$ \\
\hline \multicolumn{5}{|c|}{ CTLA-4 gene +49 A/G genotypes: } \\
\hline G/G n (\%) & $23(38.3 \%)$ & $3(15.8 \%)$ & $0.3(0.1-1.2)$ & 0.060 \\
\hline A/G n (\%) & $19(31.7 \%)$ & $11(57.9 \%)$ & $2.97(1.02-8.6)$ & 0.039 \\
\hline $\mathrm{A} / \mathrm{A} n(\%)$ & $18(30 \%)$ & $5(26.3 \%)$ & $0.8(0.3-2.6)$ & 0.500 \\
\hline \multicolumn{5}{|c|}{ CTLA-4 gene +49 A/G alleles: } \\
\hline G allele: $n(\%)$ & $65(54.2 \%)$ & $17(44.7 \%)$ & $1.2(0.4-3.8)$ & 0.500 \\
\hline A allele: $n(\%)$ & $55(45.8 \%)$ & $21(55.3 \%)$ & $3.3(0.9-12.6)$ & 0.060 \\
\hline \multicolumn{5}{|c|}{ CTLA-4 gene C/T60 genotypes: } \\
\hline $\mathrm{C} / \mathrm{C} n(\%)$ & $23(38.3 \%)$ & $13(68,4 \%)$ & $3.5(1.2-10.5)$ & 0.020 \\
\hline $\mathrm{C} / \mathrm{T} n(\%)$ & $21(35 \%)$ & $3(15.8 \%)$ & $0.3(0.1-1.3)$ & 0.090 \\
\hline $\mathrm{T} / \mathrm{T} \mathrm{n}(\%)$ & $16(26.7 \%)$ & $3(15.8 \%)$ & $0.5(0.1-2)$ & 0.300 \\
\hline \multicolumn{5}{|c|}{ CTLA-4 gene C/T60 alleles: } \\
\hline C allele: n (\%) & $67(55.8 \%)$ & $19(76.3 \%)$ & $1.9(0.5-7.6)$ & 0.300 \\
\hline T allele: $\mathrm{n}(\%)$ & $53(44.2 \%)$ & $9(23.7 \%)$ & $0.3(0.1-0.9)$ & 0.020 \\
\hline
\end{tabular}

OR: Odds Ratio Vs controls; CI: Confidence Interval; Significance was determined using Fisher's exact test

Table 4: Biochemical and hormonal parameters in different CTLA-4 $+49 \mathrm{~A} / \mathrm{G}$ polymorphism genotypes and alleles in controls and autoimmune thyroid disease cases

\begin{tabular}{|c|c|c|c|c|c|c|c|}
\hline & \multicolumn{3}{|c|}{ CTLA-4 +49 A/G genotypes } & \multirow[b]{2}{*}{$P$ value } & \multicolumn{2}{|c|}{ CTLA-4 +49 A/G alleles } & \multirow[b]{2}{*}{$P$ value } \\
\hline & $\mathrm{G} / \mathrm{G}$ & $\mathrm{A} / \mathrm{G}$ & $\mathrm{A} / \mathrm{A}$ & & $\mathrm{G}$ allele & A allele & \\
\hline Controls & $\mathrm{N}=23$ & $\mathrm{~N}=19$ & $\mathrm{~N}=18$ & & $N=65$ & $\mathrm{~N}=55$ & \\
\hline CTLA-4 (ng/mL) & $23.2 \pm 4.9$ & $25.2 \pm 4.6$ & $20.7 \pm 7.02$ & 0.06 & $23.2 \pm 4.9$ & $23 \pm 6.2$ & 0.90 \\
\hline Cholesterol (mg/dL) & $180 \pm 15.8$ & $192.3 \pm 26.8$ & $192.1 \pm 34.8$ & 0.20 & $180 \pm 15.8$ & 192.230 .5 & 0.08 \\
\hline $\mathrm{TG}(\mathrm{mg} / \mathrm{dL})$ & $137 \pm 27.5$ & $144.5 \pm 35.7$ & $157.5 \pm 39.7$ & 0.20 & $137 \pm 27.5$ & $150.8 \pm 37.8$ & 0.10 \\
\hline HDL-c (mg/dL) & $43.6 \pm 4$ & $43.9 \pm 4.9$ & $43.4 \pm 5.1$ & 0.90 & $43.6 \pm 4$ & $43.7 \pm 5$ & 0.90 \\
\hline LDL-c (mg/dL) & $109 \pm 15.2$ & $19.5 \pm 22.31$ & $117.2 \pm 29.6$ & 0.30 & $109 \pm 15.2$ & $118.4 \pm 25.8$ & 0.10 \\
\hline Free T3 (pg/mL) & $3.01 \pm 0.5$ & $3.01 \pm 0.5$ & $2.9 \pm 0.6$ & 0.80 & $3.02 \pm 0.5$ & $3 \pm 0.5$ & 0.70 \\
\hline Free T4 (ng/dL) & $0.9 \pm 0.2$ & $1.1 \pm 0.3$ & $1.1 \pm 0.2$ & 0.40 & $0.99 \pm 0.2$ & $1.1 \pm 0.2$ & 0.20 \\
\hline $\mathrm{TSH}(\mu \mathrm{IU} / \mathrm{mL})$ & $1.6 \pm 0.5$ & $2 \pm 0.8$ & $1.8 \pm 0.6$ & 0.30 & $1.6 \pm 0.5$ & $1.9 \pm 0.7$ & 0.10 \\
\hline Hypothyroidism & $\mathrm{N}=8$ & $\mathrm{~N}=20$ & $\mathrm{~N}=7$ & & $N=36$ & $N=34$ & \\
\hline CTLA-4 (ng/mL) & $73.6 \pm 10.9$ & $78.7 \pm 13.8$ & $87.1 \pm 10.1$ & 0.10 & $76.3 \pm 13.1$ & $80.2 \pm 13.1$ & 0.30 \\
\hline Cholesterol (mg/dL) & $197.1 \pm 25.1$ & $205.5 \pm 30$ & $197.6 \pm 4.7$ & 0.70 & $195.8 \pm 23.9$ & $204.2 \pm 26.3$ & 0.90 \\
\hline $\mathrm{TG}(\mathrm{mg} / \mathrm{dL})$ & $142.8 \pm 25.1$ & $157.5 \pm 21.7$ & $156.4 \pm 12.5$ & 0.20 & $148 \pm 28.3$ & $155.9 \pm 18.7$ & 0.30 \\
\hline HDL-c (mg/dL) & $38.3 \pm 4.8$ & $43.8 \pm 11.6$ & $42.3 \pm 6.5$ & 0.60 & $38.8 \pm 4.7$ & $43.4 \pm 10.6$ & 0.40 \\
\hline LDL-c (mg/dL) & $130.3 \pm 23.3$ & $130.3 \pm 30.3$ & $124 \pm 9.3$ & 0.70 & $127.4 \pm 23.4$ & $129.6 \pm 26.5$ & 0.90 \\
\hline Free T3 $(\mathrm{pg} / \mathrm{mL})$ & $1.7 \pm 0.3$ & $1.7 \pm 0.4$ & $1.5 \pm 0.6$ & 0.90 & $1.6 \pm 0.4$ & $1.6 \pm 0.4$ & 0.90 \\
\hline Free T4 (ng/dL) & $0.4 \pm 0.1$ & $0.4 \pm 0.1$ & $0.4 \pm 0.1$ & 0.90 & $0.4 \pm 0.1$ & $0.4 \pm 0.1$ & 0.80 \\
\hline $\mathrm{TSH}(\mu \mathrm{IU} / \mathrm{mL})$ & $23.5 \pm 10$ & $17.8 \pm 10$ & $16.3 \pm 4.9$ & 0.09 & $22.3 \pm 10$ & $17.6 \pm 9$ & 0.08 \\
\hline Grave's disease & $\mathrm{N}=3$ & $\mathrm{~N}=11$ & $\mathrm{~N}=5$ & & $\mathrm{~N}=17$ & $\mathrm{~N}=21$ & \\
\hline CTLA-4 (ng/mL) & $71 \pm 13.2$ & $77.6 \pm 14.8$ & $89 \pm 9.2$ & 0.10 & $71 \pm 13.2$ & $81.2 \pm 14.1$ & 0.30 \\
\hline Cholesterol (mg/dL) & $176.7 \pm 41.6$ & $197.3 \pm 47.9$ & $166 \pm 38.5$ & 0.40 & $176.7 \pm 41.6$ & $187.5 \pm 46.3$ & 0.80 \\
\hline $\mathrm{TG}(\mathrm{mg} / \mathrm{dL})$ & $130 \pm 43.6$ & $162.7 \pm 44.1$ & $118 \pm 27.7$ & 0.09 & $130 \pm 43.6$ & $148.8 \pm 44.3$ & 0.40 \\
\hline HDL-c (mg/dL) & $38.3 \pm 7.6$ & $39.5 \pm 6.5$ & $43.8 \pm 4.1$ & 0.40 & $38.3 \pm 7.6$ & $40.9 \pm 6.1$ & 0.60 \\
\hline LDL-c (mg/dL) & $112.3 \pm 41.8$ & $125.2 \pm 43.5$ & 98.633 .3 & 0.30 & $112.3 \pm 41.8$ & $116.9 \pm 41.5$ & 0.90 \\
\hline Free T3 $(\mathrm{pg} / \mathrm{mL})$ & $6 \pm 0.2$ & $6.6 \pm 0.9$ & $6.9 \pm 0.8$ & 0.30 & $6 \pm 0.2$ & $6.7 \pm 0.9$ & 0.20 \\
\hline Free T4 (ng/dL) & $3.8 \pm 0.9$ & $3.3 \pm 0.5$ & $3.1 \pm 0.7$ & 0.50 & $3.8 \pm 0.9$ & $3.2 \pm 0.6$ & 0.30 \\
\hline $\mathrm{TSH}(\mu \mathrm{IU} / \mathrm{mL})$ & $0.01 \pm 0.01$ & $0.03 \pm 0.03$ & $0.008 \pm 0.007$ & 0.60 & $0.01 \pm 0.01$ & $0.02 \pm 0.02$ & 0.80 \\
\hline
\end{tabular}

Data are given as Mean \pm SD.; CTLA-4, cytotoxic T-lymphocyte-associated antigen-4; TG, triglycerides; HDL-c, High Density Lipoprotein cholesterol; LDL-c, Low Density Lipoprotein cholesterol; T3, Tri-Iodothyronine; T4, Tetra-Iodothyronine; TSH, Thyroid Stimulating Hormone

As regards CTLA-4 gene CT60 polymorphism, the genotypes frequencies in patients without thyroid disorder (control group) were as follow: CC genotype was $(23 / 60,38.3 \%)$, CT genotype was $(21 / 60,35 \%)$ and
TT genotype was $(16 / 60,26.7 \%)$. Whereas, in patients having hypothyroidism, the $\mathrm{CC}, \mathrm{CT}$ and TT genotypes frequencies were $(21 / 35,60 \%),(7 / 35,20 \%)$ and $(7 / 35$, $20 \%$ ) respectively (Table 2 ), while in patients having 
grave's disease, the CC, CT and TT genotypes frequencies were $(13 / 19,68.4 \%),(3 / 19,15.8 \%)$ and $(3 / 19,15.8 \%)$ respectively (Table 3$)$. The CC genotype was significantly associated with developing either hypothyroidism (odd ratio: $2.4,95 \% \mathrm{CI}: 1.02-5.7, \mathrm{p}=$ 0.03 ) (Table 2) or grave's disease (odd ratio: $3.5,95 \%$ CI: $1.2-10.5, \mathrm{p}=0.02$ ) (Table 3). The odd ratio for the risk of hypothyroidism associated with the $\mathrm{T}$ allele was 0.4 (95\% CI 0.2-0.97; $\mathrm{p}=0.03$ ) (Table 2), whereas, the odd ratio for the risk of grave's disease associated with the $\mathrm{T}$ allele was 0.3 (95\% CI $0.1-0.9 ; \mathrm{p}=0.02)$ (Table 3 ). This indicates significant association of the mutant $\mathrm{T}$ allele with developing thyroid disorder in chronic HCV patients under interferon $\alpha$ therapy. However, the different genotypes and alleles distributions between control group and either hypothyroidism or grave's disease were indicative that TT genotype and the T allele are consider protective against developing thyroid disorder in chronic HCV patients under interferon $\alpha$ therapy.
The plasma CTLA-4 level was significantly increase (than controls) with presence of either hypothyroidism (P $=0.001)$ or grave's disease $(p=0.001)$ (Table 1). However, all the hormonal and biochemical parameters, including plasma CTLA-4 level, were non significantly different in different CTLA-4 gene +49 A/G polymorphism genotypes or alleles groups either in the control, hypothyroidism, or grave's disease groups (Table 4). In addition, in different CTLA-4 gene CT60 polymorphism genotypes and alleles groups in the control, hypothyroidism and grave's disease groups (Table 5), all the hormonal and biochemical parameters, including plasma CTLA-4 level, were non significantly different except that the triglycerides and free T3 were significantly different in different CT60 genotypes $(p=0.03)$ and different alleles groups $(p=$ 0.02) in hypothyroidism group.

Table 5: Biochemical and hormonal parameters in different CTLA-4 C/T60 polymorphism genotypes and alleles in controls and autoimmune thyroid disease cases

\begin{tabular}{|c|c|c|c|c|c|c|c|}
\hline & \multicolumn{4}{|c|}{ CTLA-4 genotypes } & \multicolumn{3}{|c|}{ CTLA-4 alleles } \\
\hline & $\mathrm{C} / \mathrm{C}$ & $\mathrm{C} / \mathrm{T}$ & $\mathrm{T} / \mathrm{T}$ & $P$ value & $\mathrm{C}$ allele & $\mathrm{T}$ allele & $P$ value \\
\hline Controls & $\mathrm{N}=23$ & $\mathrm{~N}=21$ & $\mathrm{~N}=16$ & & $\mathrm{~N}=67$ & $\mathrm{~N}=53$ & \\
\hline Plasma CTLA-4 (ng/mL) & $22.5 \pm 6.1$ & $23.6 \pm 5.3$ & $23.2 \pm 6.01$ & 0.80 & $22.5 \pm 6.1$ & $23.4 \pm 5.5$ & 0.50 \\
\hline Cholesterol (mg/dl) & $185.7 \pm 28.6$ & $193.3 \pm 28.6$ & $182.4 \pm 19.4$ & 0.40 & $185.8 \pm 28.6$ & $188.6 \pm 25.3$ & 0.70 \\
\hline $\mathrm{TG}(\mathrm{mg} / \mathrm{dl})$ & $149.1 \pm 34$ & $149.5 \pm 34.7$ & $135 \pm 35.4$ & 0.50 & $149.1 \pm 34$ & $143.2 \pm 35.3$ & 0.50 \\
\hline HDL-c (mg/dl) & $44.5 \pm 4.1$ & $43.5 \pm 5.6$ & $42.6 \pm 3.6$ & 0.40 & $44.5 \pm 4.1$ & $43.1 \pm 4.8$ & 0.30 \\
\hline LDL-c (mg/dl) & $111.4 \pm 24.2$ & $120 \pm 25.2$ & $112.8 \pm 15.9$ & 0.40 & $111.4 \pm 24.2$ & $116.9 \pm 21.7$ & 0.40 \\
\hline Free T3 $(\mathrm{pg} / \mathrm{mL})$ & $2.9 \pm 0.5$ & $3.1 \pm 0.5$ & $3 \pm 0.5$ & 0.70 & $2.9 \pm 0.5$ & $3.03 \pm 0.5$ & 0.40 \\
\hline Free T4 (ng/dl) & $1.1 \pm 0.2$ & $1.04 \pm 0.2$ & $1.01 \pm 0.2$ & 0.70 & $1.1 \pm 0.2$ & $1.03 \pm 0.2$ & 0.50 \\
\hline $\mathrm{TSH}(\mu \mathrm{IU} / \mathrm{mL})$ & $1.7 \pm 0.6$ & $1.8 \pm 0.6$ & $1.9 \pm 0.7$ & 0.80 & $1.7 \pm 0.6$ & $1.8 \pm 0.6$ & 0.60 \\
\hline Hypothyroidism & $\mathrm{N}=21$ & $\mathrm{~N}=7$ & $\mathrm{~N}=7$ & & $\mathrm{~N}=49$ & $\mathrm{~N}=21$ & \\
\hline Plasma CTLA-4 (ng/mL) & $78.7 \pm 9.9$ & $85 \pm 15.4$ & $75 \pm 18.4$ & 0.30 & $78.7 \pm 9.9$ & $80 \pm 17.1$ & 0.30 \\
\hline Cholesterol (mg/dl) & $201.1 \pm 20.8$ & $188.4 \pm 16.4$ & $218.1 \pm 38.4$ & 0.20 & $201.1 \pm 20.8$ & $203.3 \pm 32.3$ & 0.20 \\
\hline $\mathrm{TG}(\mathrm{mg} / \mathrm{dl})$ & $147.3 \pm 20$ & $171.9 \pm 19$ & $155.7 \pm 19.2$ & 0.03 & $147.3 \pm 20$ & $163.8 \pm 20.2$ & 0.02 \\
\hline HDL-c (mg/dl) & $41.2 \pm 8.9$ & $37.6 \pm 6.1$ & $49.9 \pm 11.03$ & 0.08 & $41.2 \pm 8.9$ & $43.7 \pm 10.7$ & 0.40 \\
\hline LDL-c (mg/dl) & $130.5 \pm 25.4$ & $116.5 \pm 16.5$ & $17.1 \pm 31.3$ & 0.20 & $130.5 \pm 25.4$ & $126.8 \pm 26.3$ & 0.30 \\
\hline Free T3 (pg/mL) & $1.7 .3 \pm 0$ & $1.3 \pm 0.5$ & $1.5 \pm 0.5$ & 0.03 & $1.7 \pm 0.3$ & $1.4 \pm 0.4$ & 0.02 \\
\hline Free T4 (ng/dl) & $0.4 \pm 0.1$ & $0.4 \pm 0.1$ & $0.4 \pm 0.1$ & 0.50 & $0.4 \pm 0.1$ & $0.4 \pm 0.1$ & 0.60 \\
\hline $\mathrm{TSH}(\mu \mathrm{IU} / \mathrm{mL})$ & $18.9 \pm 10.6$ & $15.3 \pm 3.4$ & $22 \pm 9.5$ & 0.50 & $18.9 \pm 10.6$ & $18.6 \pm 7.7$ & 0.70 \\
\hline Grave's disease & $\mathrm{N}=13$ & $N=3$ & $N=3$ & & $\mathrm{~N}=29$ & $\mathrm{~N}=9$ & \\
\hline CTLA-4 (ng/mL) & $80.8 \pm 12.1$ & $83.7 \pm 12.4$ & $70 \pm 24.2$ & 0.70 & $80.8 \pm 12.1$ & $76.8 \pm 18.8$ & 0.70 \\
\hline Cholesterol (mg/dl) & $195.4 \pm 40.1$ & $185 \pm 67.6$ & $145 \pm 26$ & 0.10 & $195.4 \pm 40.1$ & $165 \pm 50.8$ & 0.07 \\
\hline $\mathrm{TG}(\mathrm{mg} / \mathrm{dl})$ & $153.8 \pm 42.3$ & $143.3 \pm 61.1$ & $113.3 \pm 23.1$ & 0.30 & $153.8 \pm 42.3$ & $128.3 \pm 44.5$ & 0.20 \\
\hline HDL-c (mg/dl) & $39.5 \pm 6.9$ & $39.3 \pm 2.3$ & $46 \pm 1.7$ & 0.20 & $39.5 \pm 6.9$ & $42.7 \pm 4.1$ & 0.40 \\
\hline LDL-c (mg/dl) & $125.2 \pm 36.7$ & $117 \pm 57.5$ & $76.3 \pm 19.6$ & 0.10 & $125.2 \pm 36.7$ & $96.7 \pm 44.4$ & 0.07 \\
\hline Free T3 $(\mathrm{pg} / \mathrm{mL})$ & $6.7 \pm 0.9$ & $6.4 \pm 0.8$ & $6.6 \pm 0.8$ & 0.50 & $6.7 \pm 0.9$ & $6.3 \pm 0.5$ & 0.30 \\
\hline Free T4 (ng/dl) & $3.3 \pm 0.6$ & $3.2 \pm 0.9$ & $3.3 \pm 0.6$ & 0.90 & $3.3 \pm 0.5$ & $3.3 \pm 0.9$ & 0.90 \\
\hline $\mathrm{TSH}(\mu \mathrm{IU} / \mathrm{mL})$ & $0.02 \pm 0.03$ & $0.02 \pm 0.01$ & $0.02 \pm 0.02$ & 0.05 & $0.02 \pm 0.03$ & $0.01 \pm 0.01$ & 0.20 \\
\hline
\end{tabular}

Data are given as Mean \pm SD; CTLA-4, cytotoxic T-lymphocyte-associated antigen-4; TG, triglycerides; HDL-c, high density lipoprotein cholesterol; LDL-c, low density lipoprotein cholesterol; T3, tri-iodothyronine; T4, tetra-iodothyronine; TSH, thyroid stimulating hormone 


\section{DISCUSSION}

Hepatitis $\mathrm{C}$ virus is a highly immunogenic pathogen often inducing autoimmune activation changes, it can modulate $\mathrm{T}$ cell and $\mathrm{B}$ cell antibody responses, affecting many endocrine organs, most commonly the thyroid (Tran and Reeves, 2009). This is further exacerbated with the use of interferon- $\alpha$ therapy (Tran, 2010). Treatment of chronic HCV (CHC) infection with Interferon $\alpha$ (IFN) plus Ribavirin has been associated with thyroid dysfunction, in which activation of the immune system is important for the development of thyroid disease (Carella et al., 2004).

Human chromosome region $2 \mathrm{q} 33$ contains three genes known to be involved in immune regulation (Coyle et al., 2000). Two of these genes appear to positively regulate immune responses. These are the $\mathrm{CD} 28$ receptor gene and the Inducible Co-Stimulator (ICOS) gene. A third gene appears to be a negative regulator of $\mathrm{T}$ cell activation; namely, CTLA-4 (Tivol et al., 1995). It is thus not surprising that genetic variation within this region is implicated in susceptibility to autoimmune disease (Berry et al., 2008). The association of autoimmune diseases with the CTLA-4 locus could represent the effects of any of these three genes alone or in combination due to linkage disequilibrium. Ban et al., (2004) and Ueda et al. (2003) were able to show that CTLA-4 and not CD28 or ICOS was the susceptibility gene at this locus.

In addition, CTLA4 has been considered to be a permissive candidate gene involved in the etiology of autoimmune diseases; this is because CTLA-4 plays a role in the regulation of the activation of $\mathrm{T}$ cells and as well as T cell and B cell interactions (Jung et al., 2009).

Extensive population genetics studies have suggested associations between SNPs in and around CTLA-4 locus on chromosome 2 in man and the presence of autoimmune disease (Gough et al., 2005). The first of these reports was made by Yanagawa et al. (1995), who found a significant association between variation in the (AT) dinucleotide repeat within the 3'untranslated region of the CTLA-4 gene and the presence of Grave's Disease (GD). Subsequent to these findings, many others have reported associations between SNPs within and around the CLTA- 4 region and rheumatoid arthritis (Lei et al., 2005), celiac disease (Hunt et al., 2005), type I diabetes (Jung et al., 2009), myasthenia gravis (Wang et al., 2008) and autoimmune pancreatitis (Chang et al., 2007). Furthermore, the CTLA-4 gene has also been implicated in other autoimmune disorders like
Hoshimoto's Thyroiditis (HT) (Kotsa et al., 1997), Addison's disease (Vaidya et al., 2002) and Multiple sclerosis (Kouki et al., 2000).

Furthermore, the association between CTLA-4 gene and autoimmune thyroid diseases has been consistent across populations of different ethnic backgrounds, such as Caucasians (Heward et al., 1999), Japanese (Akamizu et al., 2000) and Koreans (Park et al., 2000).

Although, many studies describe the association between a polymorphism of the CTLA-4 gene and autoimmune disease, none of these previous studies was carried out in HCV patients under interferon therapy. So, the aim of this research was to analyze the frequencies of CTLA-4 CT60 and exon 1+49 A/G polymorphisms and evaluate both polymorphisms as indicators of thyroid disorder susceptibility in chronic HCV Egyptian patients under interferon therapy.

This study was carried out on 114 chronic HCV patients under combined therapy of interferon- $\alpha$ and Ribavirin. From them, 60 chronic HCV patients under combined therapy of interferon- $\alpha$ and Ribavirin without presence of thyroid disorder were considered as a control group. The other 54 patients were having thyroid disorder either hypothyroidism $(\mathrm{N}=35)$ or Grave's Disease (GD) $(\mathrm{N}=19)$. For all subjects, the genotypes of CTLA-4 gene CT60 and exon 1+49 A/G polymorphisms were studied using RFLP technique.

In this study, the $+49 \mathrm{~A} / \mathrm{G}$ genotypes frequencies in Egyptian patients without thyroid disorder (control group) were as follow: AA genotype was (30AG genotype was (31.7) and GG genotype was (38.3\%). This can be added to the results of other studies in which the distribution of genotypes ( $\mathrm{GG}, \mathrm{AG}$ and $\mathrm{AA}$ ) for CTLA-4 gene polymorphism varies among different populations including Caucasians (Yanagawa et al., 1995), Japanese (Yanagawa et al., 1997), Koreans (Park et al., 2000), Hong Kong Chinese children (Chong et al., 2008) and Taiwanese (Weng et al., 2005).

According to the SNP database of the National Center for Biotechnology Information (www.ncbi.nlm.nih.gov/SNP), the allele frequencies of exon $1+49 \mathrm{~A} / \mathrm{G}$ are very different across ethnicities. The risk $\mathrm{G}$ allele of exon $1+49 \mathrm{~A} / \mathrm{G}$ is more frequent in Asians (65than Caucasians (20) or Africans (29\%) (Wang et al., 2007b).

The present study shows that the " $G$ " allele frequency in Egyptians was 54\% in controls and $44 \%$ in GD patients. Previous studies reported that the highest "G" allele frequency $(76 \%)$ was noted in Chinese GD population (Wang et al., 2004). The lowest " $G$ " allele 
frequency $(48 \%)$ was noted in Caucasian GD population and among healthy individuals in Iranian (37\%) (Kalantari et al., 2003) and Caucasian population (38\%) (Bednarczuk et al., 2003). The study of Veeramuthumari et al. (2011), shows that the " $G$ ", allele frequency is $63 \%$ among South Indian population, which is comparable to that of the Taiwanese ' $G$ ', allele frequency (67\%) (Weng et al., 2005) and Koreans (68\%) (Park et al., 2000).

As regards the results of current research in thyroid disease patients, for CTLA-4 gene exon $1+49 \mathrm{~A} / \mathrm{G}$ polymorphism, the AG genotype was significantly increased and was significantly associated with developing thyroid disorder either hypothyroidism or grave's disease. However, the mutant $\mathrm{G}$ allele was non significantly associated with thyroid disorder. The genotype AA was decreased in patients having thyroid disorders and the odd ratio for the risk of hypothyroidism associated with the $G$ allele was 1.4 (95\% CI 0.6-3.8; $\mathrm{p}=0.3$ ), whereas, the odd ratio for the risk of grave's disease associated with the $\mathrm{G}$ allele was 1.2 (95\% CI 0.4-3.8; $\mathrm{p}=0.5$ ).

The decrease in the AA genotype and increase in the AG genotype is in accordance with previous studies on CTLA-4 gene function. It encodes a co-stimulatory molecule, which is expressed on activated $\mathrm{T}$ cells and may mediate $\mathrm{T}$ cell apoptosis (Kouki et al., 2000). In vitro studies showed that the A/G Single Nucleotide Polymorphism (SNP) at position 49 (exon 1, codon 17) of the CTLA4 gene leads to a Thr/Ala amino acid substitution in the leading peptide and is associated with impaired function of this receptor (Kouki et al., 2000). Thus CTLA-4 is a strong candidate gene for $\mathrm{T}$ cellmediated autoimmune diseases (Bednarczuk et al., 2003).

As reported previously, the distribution of CTLA-4 alleles differed significantly between control subjects and Grave's Disease (GD) patients. In both Caucasian and Japanese patients with GD (Bednarczuk et al., 2003), the frequency of $\mathrm{G}$ allele and $\mathrm{G} / \mathrm{G}$ genotype were significantly higher compared with healthy subjects. However, the relative risks for developing Graves' disease in Caucasians and Japanese conferred by $G$ allele (1.5 and 1.6 respectively) or by $G / G$ genotype (1.7 and 2.1 respectively) were different to the values found in our study $(1.2$ by $G$ allele and 0.3 by GG genotype).

In accordance with our results, studies conducted among different populations showed an association of CTLA-4 gene 49 A/G polymorphism with Graves' disease including Caucasians (Yanagawa et al., 1995), Japanese (Yanagawa et al., 1997), Koreans (Park et al.,
2000), Chinese children (Chong et al., 2008) and Taiwanese (Weng et al., 2005) and in South Indian population (Veeramuthumari et al., 2011). However, the study of Frydecka et al. (2004), showed that the distribution of CTLA-4 exon 1 A (49)G genotype, allele and phenotypic frequencies did not differ between patients with GD and healthy subjects in Polish population.

Furthermore, our study indicates that the $\mathrm{A} / \mathrm{G}$ genotype in the exon $1+49$ SNP is predominant in the Egyptian thyroid patients and that it is higher in GD patients, compared to control subjects $(\mathrm{p}=0.039)$. Compared to studies of other racial groups, our results are similar. A number of findings, including our study of association between exon $1+49$ SNP and GD, supported the theory that the exon $1+49$ SNP of CTLA-4 gene leads to genetic susceptibility to GD as a general rule (Kouki et al., 2002; Kinjo et al., 2002; Weng et al., 2005; Wang et al., 2007b).

Nithiyananthan et al. (2002), reported, in the United Kingdom patients, a significant excess of the $G$ allele in patients with autoimmune hypothyroidism compared with controls (43\% Vs $32 \%$ respectively; odds ratio 1.57) and that the AG genotype was found to be more frequent in patients with autoimmune hypothyroidism than controls (50\% Vs $46 \%$ respectively; $p=0.003)$. This is in accordance with our results (the AG genotype was significantly increase in hypothyroidism patients than control; 57.1 Vs 31.7 respectively, $\mathrm{P}=0.02$; odds ratio 2.6). In addition, Kouki et al. (2000) and Donner et al. (1997) reported a higher frequency of $\mathrm{G} / \mathrm{G}$ and $\mathrm{A} / \mathrm{G}$ genotypes and lower frequency of $\mathrm{A} / \mathrm{A}$ alleles in patients with Hashimoto thyroiditis. These previous results together with our results, suggest that the CTLA-4 gene region on chromosome $2 \mathrm{q} 33$ is a susceptibility locus for autoimmune hypothyroidism.

The fact that some control individuals bearing the G/G genotype do not develop autoimmune diseases whereas some patients with the A/A genotype suffer from autoimmunity underlines the multifactorial etiology of autoimmune diseases(Kouki et al., 2000).

Although the function of CTLA-4 protein is probably unaffected by the exon $1+49$ SNP, it may influence the level or pattern of protein expression. The $\mathrm{G}$ allele is associated with reduced control of $\mathrm{T}$ cell proliferation and thus contributes to the pathogenesis of GD, hypothyroidism and presumably of other autoimmune diseases (Kouki et al., 2000).

In the present study, we observed significantly increased serum CTLA-4 levels in GD and hypothyroidism patients compared with controls. Our results are in agreement with those of Oaks and Hallet (2000); Saverino et al. (2007) and Daroszewski et al. (2009). 
Expression of CTLA-4 appears to be selectively induced in certain pathological conditions in vivo. Ligers et al. (2001), have shown that cell-surface expression of CTLA-4 protein is influenced by common polymorphisms in the promoter and firstexon sequences. Furthermore, the effect seems to be related to the position 49 of the leader sequence (Ligers et al., 2001).

The influence of the polymorphisms within CTLA4 gene on the GD has been widely studied. The CT60 in the 3'UTR region of this gene was the most promising locus for the autoimmune thyroid disease (Daroszewski et al., 2009; Petrone et al., 2005; Weng et al., 2005).

As regards CTLA-4 gene CT60 polymorphism in current study, the genotypes frequencies in patients without thyroid disorder (control group) were as follow: CC genotype was (38.3\%), CT genotype was (35\%) and TT genotype was $(26.7 \%)$. Whereas, in patients having hypothyroidism, the $\mathrm{CC}, \mathrm{CT}$ and $\mathrm{TT}$ genotypes frequencies were $(60 \%),(20 \%)$ and $(20 \%)$ respectively, while in patients having grave's disease, the $\mathrm{CC}, \mathrm{CT}$ and TT genotypes frequencies were $(68.4 \%),(15.8 \%)$ and $(15.8 \%)$ respectively. The CC genotype was significantly associated with developing either hypothyroidism (odd ratio: $2.4,95 \% \mathrm{CI}$ : $1.02-5.7, \mathrm{p}=0.03$ ) (Table 2) or grave's disease (odd ratio: $3.5,95 \% \mathrm{CI}$ : $1.2-10.5, \mathrm{P}=$ 0.02 ). There was a significant decrease of the mutant $T$ allele with developing thyroid disorder in chronic HCV patients under interferon $\alpha$ therapy. The different genotypes and alleles distributions between control group and either hypothyroidism or grave's disease were indicative that TT genotype and the T allele are consider protective against developing thyroid disorder in chronic HCV patients under interferon $\alpha$ therapy.

The significant increase in $\mathrm{CC}$ genotype in thyroid disease patients supports that The CT60 SNP is potentially important because it may be associated with an alteration in the ratio of splice forms of the CTLA4 gene and this ratio may affect disease susceptibility, as reported by Ueda et al. (2003).

Weng et al. (2005), demonstrated that the $\mathrm{G} / \mathrm{G}$ genotype of the CT60 SNP is most prevalent in the Taiwanese population and there are more Grave's Disease (GD) patients having $G$ allele (G/G genotype) and fewer patients having $A$ allele $(A / A$ or $A / G$ genotype), compared to the control subjects. Other studies in different populations support the results of the present study (Daroszewski et al., 2009; Petrone et al., 2005; Ban et al., 2005).
In the present study, the $\mathrm{C}$ allele was increased in both GD (76.3\%) and hypothyroidism $(70 \%)$ patients than controls $(55.8 \%)$. The odd ratio for the $\mathrm{C}$ allele was 1.9 for GD and 1.5 for hypothyroidism. This is in agreement with the study of Ban et al. (2005), that reported CT60 to be most strongly associated with Autoimmune Thyroid Disease (AITD). In that study, the $\mathrm{G}$ allele of the CT60 was associated with GD than control (84.0 vs $72.6 \%, \mathrm{P}=0.0008)$ and AITD (80.1 vs. $72.6 \%, P=0.009)$ in the Japanese. Furthermore, the $G$ allele of the CT60 was associated with the increased risk for GD $[\mathrm{P}=0.004$, Odds Ratio $(\mathrm{OR})=2.0]$ and AITD $(\mathrm{P}$ $=0.03, \mathrm{OR}=1.6$ ).

In addition, according to the SNP database of the National Center for Biotechnology Information (www.ncbi.nlm.nih.gov/SNP), the risk G allele of CT60 is more common in Asians (70\%) and Africans (80\%) than Caucasians (50\%) (Wang et al., 2007b).

In the present study, the plasma CTLA-4 level was significantly increase (than controls) with presence of either hypothyroidism $(p=0.001)$ or grave's disease $(\mathrm{p}=0.001)$.

The CT60-A/G Single Nucleotide Polymorphism (SNP) is believed to control the processing and production of soluble CTLA-4 (sCTLA-4) (Purohit et al., 2005). Also, the susceptible CT60-G allele was reported to produce a lower amount of soluble CTLA- 4 mRNA in the peripheral blood lymphocytes than the disease resistant CT60-A allele (Purohit et al., 2005). These results suggested that SCTLA-4 may confer protective effect against autoimmune diseases. If this effect is true, a researcher would predict a lower sCTLA-4 in the serum in autoimmune diseases patients compared to controls. However, this prediction is in direct conflict with our results and with the observations of other studies in most autoimmune diseases including autoimmune thyroid disease (Oaks and Hallett, 2000), systemic lupus erythematosus (Liu et al., 2003) and myasthenia gravis (Wang et al., 2002b), in which the serum sCTLA-4 levels are increased in patients compared to controls.

So, elevated SCTLA-4 that has been reported in these autoimmune diseases suggests that SCTLA-4 may contribute to the development of autoimmune diseases, probably through down-regulation of $\mathrm{T}$ cell activation (Purohit et al., 2005).

In the present study, in the co-occurrence of the 2 polymorphisms (CT60 SNP and exon $1+49$ ), there are significantly more Grave's Disease (GD) and hypothyroidism patients that carry $\mathrm{G}$ alleles in the CT60 SNP and exon $1+49$ SNP, compared to control subjects. 
When the CT60 SNP genotype is G/G, the frequency of $\mathrm{A} / \mathrm{G}$ genotype in the exon $1+49 \mathrm{SNP}$ is higher. Our results confirmed that the presence of at least one $G$ allele (GG or AG) at either locus confers susceptibility to thyroid disorder. This is in agreement with results of other studies (Weng et al., 2005; Petrone et al., 2005; Chong et al., 2008). These results highlight the role of the CTLA-4 locus, in the susceptibility to GD.

The Kavvoura et al. (2007), meta-analysis study for both CT60 SNP and exon $1+49$ SNPs, examined the association of CTLA-4 polymorphisms with autoimmune thyroid disease (GD and HT) and included group-level data from more than 13,000 subjects and individual-level data from more than 5,000 subjects. The group-level data clearly showed highly significant associations of both GD and HT with each polymorphism separately (CT60 SNP and exon $1+49$ ), with $P$ values in the range of $10^{-3}$ to $10^{-16}$ in the main analyses.

Exon1 +49 A/G (21) and CT60 SNPs have been shown to have biological relevance. Both of the two SNPs have $A$ and $G$ alleles and $G$ is the risk allele for either of the two loci. Although Ueda et al. (2003), reported that CT60 is the major causal variant in determining mRNA expression, they did not find the susceptible haplotype GG carried a higher risk than single allele $\mathrm{G}$ of exon $1+49 \mathrm{~A} / \mathrm{G}$ polymorphism. This may be due to complex regulation of gene expression (for example other functional locus not considered or the discrepancy between gene expression and protein levels) (Wang et al., 2007b).

According to the SNP database of the National Center for Biotechnology Information (www.ncbi.nlm.nih.gov/SNP), the allele frequencies of exon $1+49$ A/G are very different across ethnicities. The risk $\mathrm{G}$ allele of exon $1+49 \mathrm{~A} / \mathrm{G}$ is more frequent in Asians (65\%) than Caucasians (20\%) or Africans (29\%). Similarly, the risk G allele of CT60 is more common in Asians (70\%) and Africans (80\%) than Caucasians $(50 \%)$. Therefore, the two polymorphisms provide different statistical powers for different study populations (Wang et al., 2007b).

The results of the present study revealed that plasma CTLA-4 level was not significantly different in different CTLA-4 gene $+49 \mathrm{~A} / \mathrm{G}$ or CT60 polymorphisms genotypes or alleles groups either in the control, hypothyroidism, or grave's disease groups. Thus, our data do not support a relationship between these tested SNPs and circulating levels of CTLA-4 in the presence or absence of autoimmune disease. These results are in agreement with the study of Berry et al. (2008), who found no statistically significant differences in observed versus expected genotypic frequencies for these SNPs when comparing positive versus negative blood levels of SCTLA-4.

Furthermore, our data confirm and extend the findings of Purohit et al. (2005), who reported a lack of association between CT60 genotype and sCTLA-4 levels. On the other hand, our findings appear to be at odds with the speculation that the CTLA-4 CT60-A/G SNP may determine the alternate splicing and production of the sCTLA-4 mRNA. In the Ueda et al. (2003), model, the CT60-G susceptibility allele appears to produce lower relative amounts of the SCTLA-4 mRNA; thus, one would expect that subjects at risk for autoimmune disease to have reduced levels of SCTLA-4 protein. It seems paradoxical given that lower levels of CTLA-4 message are present in susceptible individuals whereas higher levels of SCTLA-4 protein are observed in plasma of individuals with autoimmune disease (Berry et al., 2008). Possible explanations for the appearance of this discrepancy may include the possibility that there is no direct relationship between message levels at the cellular level and circulating protein in plasma. For example, elevated circulating CTLA-4 levels may simply be due to increased half-life and/or decreased turnover of protein despite increased levels of synthesis. Also, it is possible that lower levels of CTLA-4 message reflect a feedback regulatory loop in which mRNA levels are reduced in the face of higher levels of CTLA-4 protein (Berry et al., 2008).

Although the size of our samples (patients and controls) was not large, the difference in CTLA-4 genotypes frequencies between control subjects and patients was significant. Probably incorporation of both SNPs (CT60 SNP and exon $1+49$ SNP) markers further increases predictive accuracy and enhances the role of the CTLA-4 in thyroid disorders.

\section{CONCLUSION}

Our results showed that CTLA-4 gene $+49 \mathrm{~A} / \mathrm{G}$ and CT60 polymorphisms conferred susceptibility to autoimmune thyroid disorders and confirm the usefulness of the CTLA-4 genotyping in predicting thyroid disorder in chronic HCV Egyptian patients under interferon therapy.

Although researchers still cannot identify a single etiological polymorphism, our study confirms the important role of the CTLA-4 locus in determining the risk of autoimmune thyroid disorder. Furthermore, our data pointed to the possibility of using genetic screening 
to identify predisposing factors for autoimmune thyroid disorder in patients under interferon therapy.

\subsection{Recommendation}

Further large scale studies about CTLA-4 gene $+49 \mathrm{~A} / \mathrm{G}$ and CT60 polymorphisms in chronic HCV patients under interferon therapy will help to confirm results of this preliminary study in our population. Moreover, an additional power could be added by including other polymorphisms to predict thyroid disease in patients under interferon therapy. Furthermore, other follow up works are recommended in chronic HCV patients after stoppage of interferon therapy.

In addition, researchers should seek a better understanding of the underlying pathophysiology and predicting outcome of interferon therapy at its onset and identify $\mathrm{HCV}$ patients at risk of developing thyroid disorder. Such information should help to improve HCV management and allow better choosing patients for interferon therapy.

\section{REFERENCES}

Akamizu, T., M.M. Sale, S.S. Rich, H. Hiratani and J.Y. Noh et al., 2000. Association of autoimmune thyroid disease with microsatellite markers for the thyrotropin receptor gene and CTLA-4 in Japanese patients. Thyroid, 10: 851-858. DOI: 10.1089/THY.2000.10.851

Allian, C.C., L.S Poon, C.S.G. Chan, W. Richmond and P.C. $\mathrm{Fu}, 1974$. Enzymatic determination of total serum cholesterol. Clin. Chem., 20: 470-475. PMID: 4818200

Ban, Y., E.S. Concepcion, R. Villanueva, D.A. Greenberg and T.F. Davies et al., 2004. Analysis of immune regulatory genes in familial and sporadic graves' disease. J. Clin. Endocrinol. Metab., 89: 4562-4568. DOI: $10.1210 /$ jc. 2003-031693

Ban, Y., T. Tozaki, M. Taniyama, M. Tomita and Y. Ban 2005. Association of a CTLA-4 3' untranslated region (CT60) single nucleotide polymorphism with autoimmune thyroid disease in the Japanese population. Autoimmunity, 38: 151-153. DOI: 10.1080/08916930500050319

Bednarczuk, T., Y. Hiromatsu, T. Fukutani, K. Jazdzewski and P. Miskiewicz et al., 2003. Association of cytotoxic T-Lymphocyte-associated Antigen-4 (CTLA-4) gene polymorphism and non-genetic factors with Graves' ophthalmopathy in European and Japanese populations. Eur. J. Endocrinol., 148: 13-18. DOI: 10.1530/EJE.0.1480013
Berry, A., M. Tector and M.K. Oaks, 2008. Lack of association between SCTLA-4 levels in human plasma and common CTLA-4 polymorphisms. J. Negat. Results Biomed., 7: 8-8. DOI: 10.1186/14775751-7-8

Carella, C., G. Mazziotti, G. Amato, L.E. Braverman and E. Roti, 2004. Interferon- $\alpha$-related thyroid disease: Pathophysiological, epidemiological and clinical aspects. J. Clin. Endocrinol. Metab., 89: 3656-3661. DOI: $10.1210 /$ jc. 2004-0627

Chang, M.C., Y.T. Chang, Y.W. Tien, P.C. Liang and I.S. Jan et al., 2007. T-cell regulatory gene CTLA-4 polymorphism/haplotype association with autoimmune pancreatitis. Clin. Chem., 53: 17001705. DOI: 10.1373/clinchem.2007.085951

Chong, K.K.L., S.W.Y. Chiang, G.W.K. Wong, P.O.S. Tam and T.K. Ng et al., 2008. Association of CTLA-4 and IL-13 gene polymorphisms with graves' disease and ophthalmopathy in chinese children. Invest. Ophthalmol. Visual Sci., 6: 24092415. DOI: $10.1167 /$ IOVS.07-1433

Ciampolillo, A., E. Guastamacchia, L. Amati, T. Magrone and I. Munno et al., 2003. Modifications of the immune responsiveness in patients with autoimmune thyroiditis: Evidence for a systemic immune alteration. Curr. Pharm. Des., 9: 1946-1950. PMID: 12871179

Corssmit, E.P., R. Heyligenberg, E. Endert, H.P. Sauerwein and J.A. Romijn, 1995. Acute effects of interferonalpha administration on thyroid hormone metabolism in healthy men. J. Clin. Endocrinol. Metab., 80: 3140-3144. DOI: 10.1210/jc.80.11.3140

Coyle, A.J., S. Lehar, C. Lloyd, J. Tian and T. Delaney et al., 2000. The CD28-related molecule ICOS is required for effective $\mathrm{T}$ cell-dependent immune responses. Immunity. 13: 95-105. PMID: 10933398

Daroszewski, J., E. Pawlak, L. Karabon, I. Frydecka and A. Jonkisz et al., 2009. Soluble CTLA-4 receptor an immunological marker of Graves' disease and severity of ophthalmopathy is associated with CTLA-4 Jo31 and CT60 gene polymorphisms. Eur. J. Endocrinol., 161: 787-793. DOI: 10.1530/EJE-090600

Donner, H., H. Rau, P.G. Walfish, J. Braun and T. Siegmund et al., 1997. CTLA4 Alanine-17 confers genetic susceptibility to Graves' disease and to type 1 diabetes mellitus. J. Clin. Endocrinol. Metab., 82: 143-146. DOI: 10.1210/JC.82.1.143 
Dumas, B.T., W.A. Watson, H.G. Biggs, 1997. Albumin standards and the measurement of serum albumin with bromcresol green. Clin Chim Acta.258: 21-30. Doi: 10.1016/S0009-8981(96)06447-9

Fossati, P. and L. Prencipe, 1982. Serum triglycerides determined colorimetrically with an enzyme that produces hydrogen peroxide. Clin. Chem., 28: 20772080.

Friedewald, W.T., R.I. Levy and D.S. Fredrickson, 1972. Estimation of the concentration of low-density lipoprotein cholesterol in plasma, without use of the preparative ultracentrifuge. Clin. Chem., 18: 499502.

Frydecka, I., J. Daroszewski, K. Suwalska, M. Zoledziowska and A. Tutak et al., 2004. CTLA4 (CD152) gene polymorphism at position 49 in exon 1 in Graves' disease in a Polish population of the Lower Silesian region. Arch. Immunol. Ther. Exp., (Warsz)., 52: 369-374. PMID: 15507878

Furugaki, K., S. Shirasawa, N. Ishikawa, K. Ito and K. Ito et al., 2004. Association of the T-cell regulatory gene CTLA4 with Graves' disease and autoimmune thyroid disease in the Japanese. J. Hum. Genet., 49: 166-168. DOI: 10.1007/s10038-003-0120-5

Gough, S.C.L., L.S. Walker and D.M. Sansom, 2005. CTLA4 gene polymorphism and autoimmunity. Immunol. Rev., 204: 102-115. DOI: 10.1111/j.01052896.2005.00249.x

Heward, J.M., A. Allahabadia, M. Armitage, A. Hattersley and P.M. Dodson et al., 1999. The development of Graves' disease and the CTLA-4 gene on chromosome 2q33. J. Clin. Endocrinol. Metab., 84: 2398-2401. DOI: 10.1210/JC.84.7.2398

Hunt, K.A., D.P.B. McGovern, P.J. Kumar, S. Ghosh and S.P.L. Travis et al., 2005. A common CTLA4 haplotype associated with coeliac disease. Eur. J. Hum. Genet., 13: 440-444. DOI: 10.1038/sj.ejhg.5201357

Jung, M.H., J. Yu, C.H. Shin, B.K. Suh and S.W. Yang et al., 2009. Association of cytotoxic T lymphocyte antigen-4 gene polymorphisms and HLA class II alleles with the development of type 1 diabetes in korean children and adolescents. J. Korean. Med. Sci., 24: 1004-1009. DOI: 10.3346/JKMS.2009.24.6.1004

Kalantari, T., H. Mostafavi, A.M. Pezeshki, S. Farjadian and M. Doroudchi et al., 2003. Exon-1 Polymorphism of ctla-4 gene in iranian patients with Graves' disease. Autoimmunity, 36: 313-316. DOI: $10.1080 / 0891693032000114544$
Kamijo, K., K. Ishikawa and M. Tanaka, 2005. Clinical evaluation of 3 rd generation assay for thyrotropin receptor antibodies: The M22-biotin-based ELISA initiated by Smith. Endocr. J., 52: 525-529. DOI: 10.1507/ENDOCRJ.52.525

Kavvoura, F.K., T. Akamizu, T. Awata, Y. Ban and D.A. Chistiakov et al., 2007. Cytotoxic Tlymphocyte associated antigen 4 gene polymorphisms and autoimmune thyroid disease: A meta-analysis. J. Clin. Endocrinol. Metab., 92: 3162-3170. DOI: 10.1210/jc.2007-0147

Kinjo, Y., N. Takasu, I. Komiya, T. Tomoyose and M. Takara et al., 2002. Remission of Graves' hyperthyroidism and $\mathrm{A} / \mathrm{G}$ polymorphism at position 49 in exon 1 of cytotoxic $\mathrm{T}$ lymphocyte-associated molecule-4 gene. J. Clin. Endocrinol. Metab., 87: 2593-2506. DOI: $10.1210 /$ JC.87.6.2593

Kotsa, K., P.F. Watson and A.P. Weetman, 1997. A CTLA-4 gene polymorphism is associated with both Graves' disease and autoimmune hypothyroidism. Clin. Endocrinol., 46: 551-554. DOI: 10.1046/j.1365-2265.1997.1710996.x

Kouki, T., C.A. Gardine, T. Yanagawa, L.J. Degroot, 2002. Relation of three polymorphisms of the CTLA-4 gene in patients with Graves' disease. J. Endocrinol. Invest., 25: 208-213. PMID: 11936461

Kouki, T., Y. Sawai, C.A. Gardine, M.E. Fisfalen and M.L. Alegre et al., 2000. CTLA-4 gene polymorphism at position 49 in exon 1 reduces the inhibitory function of CTLA-4 and contributes to the pathogenesis of graves' disease. J. Immunol., 165: 6606-6611.

Lei, C., Z. Dongqing, S. Yeqing, M.K. Oaks and C. Lishan et al., 2005. Association of the CTLA-4 gene with rheumatoid arthritis in Chinese Han population. Eur. J. Hum. Gen., 13: 823-828. DOI: 10.1038/sj.ejhg.5201423

Ligers, A., N. Teleshova, T. Masterman, W.X. Huang and J. Hillert, 2001. CTLA-4 gene expression is influenced by promoter and exon 1 polymorphisms. Genes Immunity., 2: 145-152. DOI: 10.1038/SJ.GENE.6363752

Liu, M.F., C.R. Wang, P.C. Chen and L.L. Fung, 2003. Increased expression of soluble cytotoxic Tlymphocyte-associated antigen-4 molecule in patients with systemic lupus erythematosus. Scand. J. Immunol., 57: 568-572. DOI: 10.1046/j.13653083.2003.01232.x

Lopeo-Virella, M.F., P.G. Stone, S. Ellis and J.A. Colwell, 1977. Cholesterol determination in high density lipoproteins separated by three different methods. Clin. Chem., 23: 882-884. 
Missiha, S.B., M. Ostrowski and E.J. Heathcote, 2008. Disease progression in chronic hepatitis $\mathrm{C}$ : Modifiable and nonmodifiable factors. Gastroenterology, 134: 1699-1714. DOI: 10.1053/j.gastro.2008.02.069

Nithiyananthan, R., J.M. Heward, A. Allahabadia, J.A. Franklyn and S.C. Gough, 2002. Polymorphism of the CTLA-4 gene is associated with autoimmune hypothyroidism in the United Kingdom. Thyroid, 12: 3-6. DOI: 10.1089/105072502753451896

Oaks, M.K. and K.M. Hallett, 2000. Cutting edge: A soluble form of CTLA-4 in patients with autoimmune thyroid disease. J. Immunol., 164: 5015-5018.

Orozco, G., B. Torres, A. Nunez-Roldan, M.F. GonzalezEscribano and J. Martin, 2004. Cytotoxic Tlymphocyte antigen-4-CT60 polymorphism in rheumatoid arthritis. Tissue Antigens, 64: 667-670. DOI: 10.1111/J.1399-0039.2004.00318.X

Park, Y.J., H.K. Chung, D.J. Park, W.B. Kim and S.W. Kim et al., 2000. Polymorphism in the promoter and exon 1 of the cytotoxic T lymphocyte antigen-4 gene associated with autoimmune thyroid disease in Koreans. Thyroid, 10: 453-459. PMID: 10907987

Petrone, A., G. Giorgi, A. Galgani, I. Alemanno and S.M. Corsello et al., 2005. CT60 single nucleotide polymorphisms of the cytotoxic T-Lymphocyteassociated antigen- 4 gene region is associated with Graves' disease in an italian population. Thyroid, 15: 232-238. DOI: 10.1089/thy.2005.15.232

Purohit, S., R. Podolsky, C. Collins, W. Zheng and D. Schatz et al., 2005. Lack of correlation between the levels of soluble cytotoxic T-lymphocyte associated antigen-4 (CTLA-4) and the CT-60 genotypes. J. Autoimmune Dis., 2: 8-8. DOI: 10.1186/1740-25572-8

Saverino, D., R. Brizzolara, R. Simone, A. Chiappori and F. Milintenda-Floriani et al., 2007. Soluble CTLA-4 in autoimmune thyroid diseases: Relationship with clinical status and possible role in the immune response dysregulation. Clin. Immunol., 123: 190198. DOI: 10.1016/J.CLIM.2007.01.003

Schur, B.C., J. Bjereke, N. Nuwayhid and S.H. Wong, 2001 Genotyping of cytochrome P450 2D6*3 and *4 mutations using conventional PCR. Clin. Chem. Acta, 308: 25-31. DOI: 10.1016/S00098981(01)00422-3

Sun, T., Z. Hu, H. Shen and D. Lin, 2009. Genetic polymorphisms in Cytotoxic T-Lymphocyte Antigen 4 and cancer: The dialectical nature of subtle human immune dysregulation. Cancer. Res., 69: 6011-6011. DOI: 10.1158/0008-5472.CAN-09-0176
Thefeld, W., H. Hoffmeister, E.W. Busch, P.U. Koller and J. Vollmar, 1974. Reference values for the determination of GOT, GPT and alkaline phosphatase in serum with optimal standard methods. Dtsch Med. Wochenschr, 99: 343-344. DOI: $10.1055 / \mathrm{s}-0028-1107760$

Tivol, E.A., F. Borriello, A.N. Schweitzer, W.P. Lynch and J.A. Bluestone et al., 1995. Loss of CTLA-4 leads to massive lymphoproliferation and fatal multiorgan tissue destruction, revealing a critical negative regulatory role of CTLA-4. Immunity, 3: 541-547. DOI: 10.1016/1074-7613(95)90125-6

Torres, B., F. Aguilar, E. Franco, E. Sanchez and J. Sanchez-Roman et al., 2004. Association of the CT60 marker of the CTLA4 gene with systemic lupus erythematosus. Arthritis Rheum., 50: 22112215. DOI: $10.1002 /$ art.20347

Tran, H.A. and G.E. Reeves, 2009. The influence of hepatitis $\mathrm{C}$ infection and interferon-alpha therapy on thyrotropin blocking and stimulating autoantibodies in Graves' ophthalmopathy: A case report. Thyroid Res., 2: 12-12. PMID: 19954547

Tran, H.A., 2010. The swinging thyroid in hepatitis C infection and interferon therapy. Q.J.M., 103: 187191. DOI: $10.1093 /$ qjmed/hcp 157

Ueda, H., J.M. Howson, L. Esposito, J. Heward and H. Snook et al., 2003. Association of the T-cell regulatory gene CTLA4 with susceptibility to autoimmune disease. Nature, 423: 506-511. DOI: 10.1038 /nature 01621

Vaidya, B. and S. Pearce, 2004. The emerging role of the CTLA-4 gene in autoimmune endocrinopathies. Eur. J. Endocrinol., 150: 619-626. DOI: 10.1530/EJE.0.1500619

Vaidya, B., S.H.S. Pearce, S. Charlton, N. Marshall and A.D. Rowan et al., 2002. An association between the CTLA4 exon 1 polymorphism and early rheumatoid arthritis with autoimmune endocrinopathies. Rheumatology, 41: 180-183. DOI: 10.1093/rheumatology/41.2.180

Vanderpump, M.P., W.M. Tunbridge, J.M. French, D. Appleton and D. Bates el al., 1995. The incidence of thyroid disorders in the community: A twenty-year follow-up of the Whickham Survey. Clin. Endocrinol., 43: 55-68. DOI: 10.1111/J.13652265.1995.TB01894.X

Veeramuthumari, P., W. Isabel and K. Kannan, 2011. A study on the level of $\mathrm{T}_{3}, \mathrm{~T}_{4}$, TSH and the association of A/G polymorphism with CTLA-4 gene in graves' hyperthyroidism among South Indian population. Ind. J. Clin. Biochem., 26: 66-69. 
Wang, L., D. Li, Z. Fu, H. Li and W. Jiang et al., 2007a. Association of CTLA-4 gene polymorphisms with sporadic breast cancer in Chinese Han population. BMC Cancer. 7: 173-173. DOI: 10.1186/1471-24077-173

Wang, P.W., I.Y. Chen, R.T. Liu, C.J. Hsieh and E. Hsi et al., 2007b. Cytotoxic T lymphocyte-associated molecule-4 gene polymorphism and hyperthyroid Graves' disease relapse after antithyroid drug withdrawal: A follow-up study. J. Clin. Endocrinol. Metab., 92: 2513-2518. DOI: 10.1210/jc.2006-2761

Wang, P.W., R.T. Liu, S.H.H. Jou, S.T. Wang and Y.H. $\mathrm{Hu}$ et al., 2004. Cytotoxic T lymphocyte-associated molecule-4 polymorphism and relapse of Graves' hyperthyroidism after antithyroid withdrawal. J. Clin. Endocrinol. Metab., 89: 169-73. DOI: 10.1210/JC.2003-030854

Wang, S.H., J.D. Bretz, E. Phelps, E. Mezosi and P.L. Arscott et al., 2002a. A unique combination of inflammatory cytokines enhances apoptosis of thyroid follicular cells and transforms nondestructive to destructive thyroiditis in experimental autoimmune thyroiditis. J. Immunol., 168: 2470-2474. PMID: 11859140

Wang, X.B., M. Kakoulidou, R. Giscombe, Q. Qiu and D. Huang et al., 2002b. Abnormal expression of CTLA-4 by $\mathrm{T}$ cells from patients with myasthenia gravis: Effect of an AT-rich gene sequence. J. Neuroimmunol., 130: 224-232. DOI: $10.1016 / \mathrm{S} 0165-5728(02) 00228-\mathrm{X}$
Wang, X.B., R. Pirskanen, R. Giscombe and A.K. Lefvert, 2008. Two SNPs in the promoter region of the CTLA-4 gene affect binding of transcription factors and are associated with human myasthenia gravis. J. Internal Med., 263: 61-69. DOI: $10.1111 /$ j.1365-2796.2007.01879.x

Weng, Y.C., M.J. Wu and W.S. Lin, 2005. CT60 single nucleotide polymorphism of the CTLA-4 gene is associated with susceptibility to Graves' disease in the Taiwanese population. Ann. Clin. Lab. Sci., 35: 259-264. PMID: 16081581

Yanagawa, T., M. Taniyama, S. Enomoto, K. Gomi and H. Maruyama et al., 1997. CTLA4 gene polymorphism confers susceptibility to Graves' disease in Japanese. Thyroid. 7: 843-846. DOI: 10.1089/THY.1997.7.843

Yanagawa, T., Y. Hidaka, V. Guimaraes, M. Soliman and L.J. DeGroot, 1995. CTLA-4 gene polymorphism associated with Graves' disease in a Caucasian population. J. Clin. Endocrinol. Metab., 80: 41-45. DOI: 10.1210/jc.80.1.41 